\title{
ASOCIACIÓN DE TRASTORNOS NUTRICIONALES CON ALTERACIONES DEL GUSTO Y EL ARDOR DE LA MUCOSA ORAL EN LAS PERSONAS MAYORES RESIDENTES EN SAN ANDRÉS (SANTANDER)
}

\author{
${ }^{1}$ Lizeth Johana Muñoz Pérez, ${ }^{1}$ Diana Riátiga Ortiz, ${ }^{2}$ Sonia Constanza Concha Sánchez \\ ${ }^{1}$ Odontóloga F. de Odontología U. Santo Tomás, Bucaramanga (Colombia) \\ ${ }^{2}$ Odontóloga U. Santo Tomás, Especialista en Educación y Comunicación para la Salud y el Bienestar U. Industrial de Santander, \\ Magíster en Epidemiología U. Industrial de Santander, Candidata a Doctor U. Nacional de Colombia, Docente U. Santo Tomás, Bucaramanga (Colombia).
}

Autor responsable de correspondencia: Sonia Constanza Concha Sánchez

Correo electrónico: sococosa@yahoo.com

RESUMEN

Objetivo: determinar una posible asociación entre el ardor de la mucosa oral y la disgeusia con alteraciones nutricionales en las personas mayores.

Materiales y métodos: se realizó un estudio observacional analítico de corte transversal, el universo y la muestra lo constituyeron 46 personas institucionalizadas y 44 no institucionalizadas mayores de 50 años. Se estudiaron variables sociodemográficas, parámetros nutricionales, ardor bucal y disgeusia. Se calcularon medidas de resumen en el análisis univariado; para el bivariado se consideró como variable de salida la condición nutricional y se aplicó el Chi ${ }^{2}$ o test Exacto de Fisher para variables cualitativas y para las cuantitativas la prueba ANOVA o Kruskal Wallis. Se aplicó un análisis de regresión binomial que estimó las Razones de Prevalencia (RP) y sus respectivos intervalos de confianza. Se consideró un nivel de significancia de alfa $(\alpha)=0,05$.

Resultados: el ardor bucal no se asoció con las alteraciones nutricionales. La alteración en la percepción del sabor amargo se comportó como un factor protector con RP 0,7 (IC 95\% 0,3 - 0,9). El 34,4\% reportó sufrir de ardor bucal y el 73,3\% registró disgeusia. Las variables que se asociaron con el estado desnutrición o riesgo a desnutrición según el Mini Nutritional Assessment (MNA) fueron vive independiente (RP 8,3 IC 95\% 1,8 83,3), dificultad para masticar y boca seca ambos con RP 1,4 (IC 95\% 1,2 1,7).

Conclusión: la condición nutricional en los adultos mayores se asoció con la dependencia que registraban los adultos mayores, la dificultad para masticar y la boca seca. La alteración en la percepción del sabor amargo se comportó como factor protector. [Muñoz LJ, Riatiga $\mathrm{D}$, Concha SC. Asociación de trastornos nutricionales con alteraciones del gusto y el ardor de la mucosa oral en las personas mayores residentes en San Andrés (Santander). Ustasalud 2012; 11: 19 - 31]

Palabras clave: Desnutrición, Disgeusia, Ardor bucal, Adulto mayor.

\section{ASSOCIATION BETWEEN NUTRITIONAL DISORDERS WITH BURNING OF THE ORAL MUCOSA AND DYSGEUSIA IN THE ELDERLY RESIDENTS IN SAN ANDRES (SANTANDER)}

\begin{abstract}
Objective: to analyze the association of the burning of the oral mucosa and dysgeusia with nutritional disorders in the elderly. Methods: an observational analytic cross sectional study was done. The universe and the sample consisted of 46 institutionalized and 44 non-institutionalized older people aged 50 or more residents in San Andrés (Santander). We analyzed sociodemographic variables, nutritional parameters, burning mouth and dysgeusia. The univariate statistical analysis plan included the calculation of summary measures, for the bivariate considered as output variable nutritional status, were applied chi ${ }^{2}$ test or Fisher exact test for qualitative variables and ANOVA for quantitative test and Kruskal Wallis. For the multivariate analysis was applied a binomial regression analysis, which estimated the RR (relative risk ratio of prevalence) and their respective confidence intervals for the entire analysis is considered a significance level of alpha $(\alpha)=0.05$.

Results: the burning mouth was not associated with nutritional disorders in the elderly evaluated. The alteration in the perception of bitter taste behaved as a protective factor with PR 0,7 (CI 95\% 0.3 0.9). The 34.4\% of those evaluated reported suffering from burning mouth. $73.3 \%$ of all seniors reported dysgeusia and $43.3 \%$ of them (39) reported alteration in the perception of sweetness. Twenty percent (18) of seniors reported malnutrition and $34.4 \%$ (31) at risk of malnutrition according to what was registered by the MNA. Variables that were associated with the state of malnutrition or malnutrition risk (according to MNA) were living independently (PR 8.3 CI 95\% 1.8 83.3) and factors within the oral difficulty chewing and dry mouth with both (PR 1.4 CI 95\% 1.2, 1.7).

Conclusion: the nutritional status in older adults was associated with the unit that recorded the elderly, difficulty chewing and dry mouth. The alteration in the perception of bitter taste behaved as a protective factor.
\end{abstract}

Key words: Malnutrition, Dysgeusia, Burning mouth, Elderly, Geriatric.

Recibido para publicación: 10 de mayo de 2012. Aceptado para publicación: 11 de junio de 2012. 


\section{INTRODUCCIÓN}

La cavidad oral es asiento de una diversidad de patologías de origen tanto local como sistémico por lo que se desarrollan lesiones de carácter multifactorial y donde los factores psicógenos constituyen una importante variable para ser considerada. ${ }^{1} \mathrm{La}$ relación se fundamenta en el principio de que la cavidad bucal no sólo es la vía de alimentación y de comunicación, sino también una zona primaria de placer que puede reflejar somatizaciones psicológicas. Esta relación puede influir de forma significativa en la percepción de calidad de vida por parte del paciente y modificar la ingesta de alimentos en las personas mayores. ${ }^{1-3}$

A pesar de que el ardor bucal, la disgeusia (alteración del gusto) y las deficiencias nutricionales han sido ampliamente estudiadas en forma independiente, su etiología es incierta y poco se ha analizado del potencial impacto del ardor bucal y la disgeusia sobre la condición nutricional. Sin embargo, se sugieren múltiples factores de origen local, sistémico y psicológico como posibles agentes etiológicos. A menudo la interacción de estos factores es compleja y no se sabe si son los que originan los síntomas, o bien son los síntomas los que determinan el perfil característico de los pacientes con este tipo de trastornos. ${ }^{1-5}$

Con esta investigación se pretende despertar interés en los profesionales de la salud, especialmente en odontólogos, acerca de la posible relación entre el ardor bucal incluida la pérdida del gusto con el estado nutricional y cómo estos factores afectan la salud del paciente geriátrico. De esta manera, se podrían generar programas de promoción y prevención que garanticen una mejor calidad de vida en las personas de la tercera edad.

El ardor bucal, es un trastorno intrabucal doloroso que no conlleva signos clínicos aparentes y se caracteriza por sensación de ardor en la cavidad bucal, observándose la mucosa normal. El término 'ardor bucal' se emplea cuando hay disestesias bucales en presencia de una mucosa clínicamente normal. ${ }^{1,3,4}$ No obstante, muchos autores lo justifican ante la evidencia que la sensación de quemazón con frecuencia se manifiesta junto con xerostomía, pérdida o alteración del gusto, sensación de intolerancia a prótesis dentarias y síntomas en otras partes del cuerpo, como artralgias, dolor facial atípico y odontalgia atípica. Se puede considerar que el 'ardor bucal' es un síntoma que se manifiesta en determinados tipos de lesiones bucales y en el Síndrome de Boca Urente (SBU) ${ }^{6,7}$ La causa del ardor bucal es desconocida. Sin embargo, se ha identificado una compleja asociación de factores biológicos y psicológicos que hacen suponer una etiología multifactorial. ${ }^{3,8.9}$

Muchos estudios han documentado la prevalencia de malnutrición en adultos mayores, tanto institucionalizados como no institucionalizados. La prevalencia va desde el $10 \mathrm{al} 20 \%$ en adultos mayores en sus hogares, $60 \%$ en pacientes hospitalizados y en instituciones con cuidados especiales. Diferentes situaciones y factores (ambientales, funcionales, psicológicos, nutricionales y médicos) pueden llevar a que el adulto mayor disminuya su ingesta de alimentos lo que contribuye al desarrollo de malnutrición. ${ }^{10-12}$

En adultos mayores institucionalizados el estado nutricional se ve afectado principalmente por factores relacionados con la ingesta puesto que la desnutrición se asocia positivamente con disfagia, consumo lento de alimentos, ingesta proteica baja, apetito disminuido, presencia de sonda para la alimentación y la edad. ${ }^{10-12}$

La evaluación nutricional en el anciano es especialmente difícil porque muchos de los signos relacionados con la desnutrición lo están también con el proceso de envejecimiento. Es tan frecuente la presencia de desnutrición en algún grado que se debe tener en mente cada vez que se atienda a personas mayores con factores de riesgo (que vivan solas, que presente cierto deterioro cognitivo, que haya perdido peso últimamente, que presente capacidad económica limitada) tanto de forma aislada como asociada a otras patologías (frecuentemente asociada a demencias leves). ${ }^{13,14}$ Actualmente, se utiliza una evaluación del estado nutricional que incorpora parámetros funcionales, dietéticos y subjetivos denominados Mini Nutritional Assessment (MNA) que en español significa Valoración Nutricional Mínina y que ha sido validado en varios estudios. ${ }^{10,15,16}$

Por otra parte, las alteraciones del gusto se clasifican en dos grandes grupos: cualitativas y cuantitativas. ${ }^{17,18}$ La alteración cualitativa del gusto más habitual es la disgeusia, que consiste en una sensación gustativa extraña o distorsionada ante estímulos convencionales. ${ }^{17}$

Los pacientes con pérdida del sentido del gusto deben ser evaluados en lo que se refiere a las funciones olfatoria y gustativa al realizar pruebas para determinar el umbral de la percepción de los diferentes sabores y olores. Hay que destacar que las pruebas para la determinación de la función gustativa están menos estandarizadas que las correspondientes a los olores. Para determinar los sabores básicos, se utiliza una prueba de discriminación triangular en lugar de la prueba convencional de determinación 
de umbrales porque con la prueba de discriminación se determina la existencia o no de diferencias respecto a un sabor neutro, el del agua, lo que hace que los resultados sean más objetivos. ${ }^{19}$ Además, se evidencia qué alteración en la capacidad de percibir sabores puede afectar el consumo normal de alimentos por parte de las personas mayores y traer consigo trastornos nutricionales y alteraciones en la calidad de vida de las personas. Por lo tanto, se consideró importante analizar la asociación que tiene el ardor de la mucosa y la disgeusia con alteraciones nutricionales en las personas mayores residentes en el municipio de San Andrés en Santander.

\section{MATERIALES Y MÉTODOS}

Se realizó un estudio observacional analítico de corte transversal. El universo y la muestra la conformaron 90 personas mayores de 50 años pertenecientes a un hogar geriátrico y a un grupo de la tercera edad del municipio de San Andrés (Santander) distribuidos así: 46 personas pertenecían al hogar geriátrico y 44 estaban vinculados al grupo de la tercera edad existente en el municipio mencionado.

Como criterios de inclusión se consideraron ser adultos mayores de 50 años pertenecientes al hogar geriátrico y al grupo de la tercera edad del municipio de San Andrés (Santander). No se incluyeron personas con trastornos mentales, incapacidad motriz severa, individuos con problemas de esquizofrenia según historia clínica y negación por parte de la persona al examen clínico.

En esta investigación se aplicó un instrumento en el que se registraron variables sociodemográficas (edad, género, procedencia, vinculación, nivel de escolaridad) variables sobre la Valoración Nutricional Mínima (MNA), ${ }^{15,20,21}$ variables para la valoración del ardor bucal; sensación de boca seca, nivel de ardor en la boca, medicamentos, tipo de medicamentos, dificultad para masticar los alimentos, presencia de prótesis dentales, prótesis desadaptadas, presencia de candidiasis, sensación de boca seca y flujo salival. ${ }^{2}$ Por último, se tuvo en cuenta dentro de las variables para la disgeusia: autorreporte de percepción de sabores, percepción de sabor salado, ácido, amargo, dulce, percepción de alteración de alguno de los sabores. ${ }^{19}$

Previa a la recolección de datos se efectuó una prueba piloto con una población no institucionalizada (6 personas) con características similares a la población evaluada. Esta prueba piloto sirvió para realizar modificaciones en las encuestas, bases y pruebas estadística aplicadas en pacientes residentes de Bucaramanga, a partir de ésta se modificó el instrumento, se estandarizaron los procedimientos y se definieron los tiempos para el desarrollo de la investigación y el diligenciamiento de la encuesta.

Se recolectaron variables sociodemográficas mediante un proceso de encuesta en el que se interrogaban a la persona mayor involucrada. Para la recolección de las variables generales se valoró mediante observación, las variables relacionadas con movilidad y úlceras y lesiones de la piel y las relacionadas con problemas neuropsicológicos y condición sistémica se recolectarán de la historia clínica del paciente.

Para efectuar la valoración nutricional se aplicó parámetros relacionados con la encuesta MNA, se efectuaron los siguientes procedimientos se procedió a la toma de talla y peso y demás medidas antropométricas. Para analizar aspectos relacionados con el ardor bucal se interrogó y se realizó una valoración oral en las personas mayores incluida la toma de muestra de saliva estimulada, además se interrogó y se realizó valoración oral en las personas mayores de aspectos que tenían, se analizó presencia e intensidad de ardor en la boca recolectada mediante escala análoga visual, además, se recolectó consumo de medicamentos, presencia y nivel de adaptación de las prótesis dentales.

Para las variables relacionadas con parámetros dietéticos se procedió a interrogar al paciente y se corroboró con la persona que suministraba la alimentación para verificar la consistencia de la información reportada. Para los criterios que tenían que ver con la valoración subjetiva de percepción del estado nutricional se interrogó a la persona mayor y para la consolidación de la valoración nutricional se aplicaron los criterios definidos para este índice. ${ }^{15}$

Para los aspectos relacionados con la valoración de la disgeusia se procedió a interrogar y finalmente a efectuar la valoración objetiva para los diferentes sabores de la siguiente manera: se valoraron los cuatro estímulos clásicos del gusto (dulce, salado, ácido y amargo). Para determinar los sabores básicos se optó por utilizar una prueba de discriminación triangular. La persona probaba estas tríadas en las que la muestra diferente varía en cada una y aumenta paulatinamente la concentración de dichas sustancias. Para cada uno de los estímulos ya mencionados se dispuso de tres concentraciones: baja $(0,05 \mathrm{ml} / 100 \mathrm{ml}$ de agua), media $(0,1 \mathrm{ml} / 100 \mathrm{ml}$ de agua) y alta $(0,5 \mathrm{ml} / 100 \mathrm{ml}$ de agua). Ejemplo: poco dulce, dulce y muy dulce. ${ }^{19}$

Los datos se procesaron en Excel por duplicado, se exportaron a EPI-INFO 6.04 y mediante la rutina valídate se corroboró la calidad de la digitación. Finalmente, se realizaron los análisis en el paquete estadístico STATA 9.0. El análisis univariado incluyó 
las variables mediante medidas de tendencia central y dispersión según su naturaleza y el bivariado se estableció la relación entre la condición con cada variable a través del test de $\mathrm{Chi}^{2} \mathrm{y}$ el test exacto de Fischer para las variables cualitativas; para las variables cuantitativas se aplicaron pruebas de $t$ de Student o de rangos de Wicolxon según la distribución de los datos. ${ }^{21}$

Para el análisis multivariado, se aplicó una regresión binomial, que estimó las Razones de Prevalencia (RP) y sus respectivos intervalos de confianza. Se definió como variable de salida la condición nutricional lo que implicó recategorizar la variable al definir como evento a aquellos que de acuerdo con el MNA se catalogaron con desnutrición o a riesgo de desnutrición y como libres del evento a los que el MNA clasificó como bien nutridos. Las variables explicatorias fueron las sociodemográficas, los factores que pudiesen condicionar el consumo de alimentos y la condición nutricional y finalmente, los relacionados con ardor bucal y disgeusia. La regresión binomial no ajustada, implicó un análisis bivariado en el que se relacionó la variable de salida con cada una de las explicatorias, en forma independiente; a partir de este, se seleccionaron las variables para el modelo final si su valor de $p$ fue menor o igual a 0,20 ( $p$ $\leq 0,20$ ) y se forzaron en el modelo aquellas que se consideraron biológicamente importantes. A partir de éstas se obtuvo el modelo de regresión binomial ajustado. $^{21}$
Los principios éticos básicos que se adoptaron al momento de realizar esta investigación fueron: el respeto a la autonomía, no maleficencia, beneficencia, justicia y confidencialidad al tratar siempre de proteger la integridad del anciano de acuerdo con la resolución 008430 de 1993 emitida por el Ministerio de Salud de la República de Colombia. ${ }^{22}$

\section{RESULTADOS}

\section{Análisis univariado}

\section{Variables sociodemográficas}

Se evaluaron 90 adultos mayores. El 58,9\% (53) eran mujeres; el promedio de edad en la población analizada fue de 70,4 $\pm 8,6$ años. El 98,9\% (89) procedían de la zona urbana, el $51,1 \%$ (46) estaba vinculado al hogar geriátrico María Rafols y el 58,9\% (53) reportaban tener un nivel educativo primario (Tabla 1).

\section{Variables valoración antropométrica MNA}

La talla promedio en la población evaluada fue de $1,57 \pm 0,1$ metros y el peso de 58,2 $\pm 12,3 \mathrm{Kg}$. El $52,2 \%$ (47) presentó un Índice de Masa Corporal (IMC) $>23 \mathrm{Kg} / \mathrm{m}^{2}$. El 71,1\% (64) registró una circunferencia braquial mayor a $22 \mathrm{~cm}$, el 91,1\% (84) tenía una circunferencia de pierna mayor a $31 \mathrm{~cm}$ y finalmente, el 70\% (63) de la población no sabía si había reportado pérdida de peso los últimos tres meses (Tabla 2).

Tabla 1. Descripción de la condición nutricional según las variables socio-demográficas analizadas en la población adulta mayor del municipio de San Andrés (Santander) recolectadas en 2009

\begin{tabular}{|c|c|c|c|c|c|}
\hline Variable & $\begin{array}{l}\text { Global } \\
\text { n (\%) }\end{array}$ & $\begin{array}{c}\text { Desnutrido } \\
\text { n (\%) }\end{array}$ & $\begin{array}{c}\text { Riesgo de } \\
\text { desnutrición } \\
\text { n (\%) }\end{array}$ & $\begin{array}{c}\text { Bien nutrido } \\
\text { n (\%) }\end{array}$ & $\mathbf{P}^{*}$ \\
\hline Población & $90(100 \%)$ & $18(20.00)$ & $31(34.44)$ & 41 (45.56) & \\
\hline Edad (años) & $70,4 \pm 8,6$ & $73,8 \pm 8,3$ & $73,7 \pm 8,2$ & $66,5 \pm 7,4$ & $0,002^{* * *}$ \\
\hline Género & & & & & $0,010^{*}$ \\
\hline Femenino & $53(58,9)$ & $7(13,2)$ & $15(28,3)$ & $31(58,5)$ & \\
\hline Masculino & $37(41,1)$ & $11(29,7)$ & $16(43,2)$ & $10(27,2)$ & \\
\hline Procedencia & & & & & $0,200^{* *}$ \\
\hline Urbana & $89(98,9)$ & $17(19,1)$ & $31(34,8)$ & $41(46,1)$ & \\
\hline Rural & $1(1,1)$ & $1(100,0)$ & $0(0,0)$ & $0(0,0)$ & \\
\hline Vinculación & & & & & $<0,0001^{* *}$ \\
\hline No institucionalizado & $44(48,9)$ & -- & $3(6,8)$ & $41(93,2)$ & \\
\hline Institucionalizado & $46(51,1)$ & $18(39,1)$ & $28(60,9)$ & $0(0,0)$ & \\
\hline Nivel de escolaridad & & & & & $<0.0001^{* *}$ \\
\hline Ninguno & $31(34,4)$ & $13(41,9)$ & $15(48,4)$ & $3(9,7)$ & \\
\hline Primaria & $53(58,9)$ & $5(9,4)$ & $16(30,2)$ & $32(60,4)$ & \\
\hline Secundaria & $5(5,6)$ & -- & -- & $5(100,0)$ & \\
\hline Otros & $1(1,1)$ & -- & -- & $1(100,0)$ & \\
\hline
\end{tabular}

$\alpha=0.05^{*}$ Test Chi $^{2 *}$ Test exacto Fisher ${ }^{* * *}$ ANOVA 
Tabla 2. Descripción de la condición nutricional considerando valoración antropométrica MNA

\begin{tabular}{|c|c|c|c|c|c|}
\hline Variable & $\begin{array}{l}\text { Global } \\
\text { n (\%) }\end{array}$ & $\begin{array}{c}\text { Desnutrido } \\
\mathbf{n}(\%)\end{array}$ & $\begin{array}{c}\text { Riesgo de } \\
\text { desnutrición } \\
\text { n (\%) }\end{array}$ & $\begin{array}{c}\text { Bien nutrido } \\
\text { n (\%) }\end{array}$ & $\mathbf{P}^{*}$ \\
\hline Talla & $1,57 \pm 0,1$ & $1,56 \pm 0,1$ & $1,56 \pm 0,1$ & $1,59 \pm 0,07$ & $0,1041^{* * *}$ \\
\hline Peso (Kgs) & $58,2 \pm 12,3$ & $47,4 \pm 10,6$ & $56,0 \pm 9,7$ & $64,8 \pm 11,1$ & \\
\hline Índice de Masa Corporal & & & & & $<0,0001^{* * *}$ \\
\hline Menor de 19 & $10(11,1)$ & $4(40,0)$ & $4(4,0)$ & $2(20,0)$ & \\
\hline De 19 a 21 & $18(20,0)$ & $10(55,6)$ & $7(46,7)$ & $4(26,7)$ & \\
\hline De 22 a 24 & $15(16,7)$ & $4(26,7)$ & $7(46,7)$ & $4(26,7)$ & \\
\hline Mayor de 24 & $47(52,2)$ & -- & $15(31,9)$ & $32(68,1)$ & \\
\hline Circunferencia braquial (cm) & & & & & $<0,0001^{* *}$ \\
\hline Menor de 21 & $4(4,4)$ & $2(50,0)$ & $2(50,0)$ & -- & \\
\hline De 21 a 22 & $22(24,4)$ & $14(63,6)$ & $6(27,03)$ & $2(9,1)$ & \\
\hline Mayor de 22 & $64(71,1)$ & $2(3,1)$ & $23(35,9)$ & $39(60,9)$ & \\
\hline Circunferencia de la pierna $(\mathrm{cm})$ & & & & & $0,052^{*}$ \\
\hline Menor de 31 & $6(6,7)$ & $4(66,7)$ & $1(16,7)$ & $1(16,7)$ & \\
\hline Mayor de 31 & $82(91,1)$ & $14(17,1)$ & $30(36,6)$ & $38(46,3)$ & \\
\hline Pérdida de peso & & & & & $<0,0001^{* *}$ \\
\hline De 1 a $3 \mathrm{Kg}$ & $5(5,6)$ & -- & $2(40,0)$ & $3(60,0)$ & \\
\hline Mayor de $3 \mathrm{Kg}$ & $2(2,2)$ & -- & $100(6,5)$ & -- & \\
\hline No sabe & $63(70,0)$ & $18(28,6)$ & $27(42,8)$ & $18(28,6)$ & \\
\hline Sin pérdida & $20(22,2)$ & -- & -- & $20(100,0)$ & \\
\hline
\end{tabular}

\section{Variables correspondientes a valoración global del MNA}

El 50\% (45) de la población manifestó vivir en forma independiente, el $72,2 \%$ (65) no consumía más de tres medicamentos; el 73,3\% (66) no sufría estrés psicológico y sólo el $2,2 \%$ (2) reportó no poder levantarse ni de la cama ni de la silla en la que se encuentre. El 61,1\% (55) no registró demencia y el $96,7 \%$ (57) no presentaba lesiones en la piel (Tabla 3).

\section{Variables correspondientes a valoración dietética y subjetiva}

El 98,9\% (89) de la población reportó consumir sus alimentos tres veces al día; el $42,2 \%$ (38) manifestó consumir dos proteínas diarias; el 91,11\% (82) comía alguna fruta o verdura al día. El 56,7\% (51) de los sujetos reportó una pérdida moderada del apetito y el 67,8\% (61) registró consumir menos de tres tazas de líquidos al día. El 78,9\% (71) manifestó no tener dificultades para autoalimentarse.

El 62,2\% (56) de las personas percibía una alteración nutricional moderada y al comparar su nivel nutricional con otras personas el 38,9\% (35) reconocía no saber cómo era su estado nutricional (Tabla 4).
Variables relacionadas con Síndrome de Boca Urente (SBU) y otros factores orales

El 34,4\% (31) reportó tener ardor en su boca con un promedio en el nivel de ardor de 1,8 $\pm 2,6 \mathrm{~cm}$. El $81,1 \%$ (73) registró medicación, el 44,4\% (40) dificultades masticatorias, el 76,7\% (69) presencia de prótesis pero sólo el $31,11 \%$ (28) presentaba prótesis inferior. El 16,7\% (15) registró prótesis desadaptadas y la presencia de cándida en igual cantidad de sujetos, el 55,6\% (50) reportó tener boca seca y el 34,4\% (31) presentaba un flujo salival anormal (Tabla 5).

\section{Variables relacionadas con tipo de medicamentos}

El promedio de medicamentos consumidos por los sujetos evaluados fue de $1,7 \pm 1,1$. El 56,7\% (51) consumía antihistamínicos, el 28,9\% (26) diuréticos, el 15,6\% (14) hipoglicemiantes, el 17,8\%(16) tomaba ansiolíticos y corticoesteroides (Tabla 6).

\section{Variables relacionadas con disgeusia}

El 96,7\% (87) de los adultos mayores autoreportaron alteración en la percepción de los sabores; al análisis organoléptico el $73,3 \%$ (66) registraron disgeusia, el $43,3 \%$ (39) de las personas no percibían los sabores dulces y el 23,3\% (21) los sabores amargos, sólo el $17,8 \%$ (16) no percibían los sabores ácidos (Tabla 7). 
ARTÍCULO ORIGINAL

Tabla 3. Descripción de condición nutricional al considerar la valoración global del MNA

\begin{tabular}{|c|c|c|c|c|c|}
\hline Variable & $\begin{array}{c}\text { Global } \\
\text { n (\%) }\end{array}$ & $\begin{array}{c}\text { Desnutrido } \\
\text { n (\%) }\end{array}$ & $\begin{array}{c}\text { Riesgo de desnutrición } \\
\mathrm{n}(\%)\end{array}$ & $\begin{array}{c}\text { Bien nutrido } \\
\mathrm{n}(\%)\end{array}$ & $\mathbf{P}^{*}$ \\
\hline Vivo independiente & & & & & $<0,0001^{* * *}$ \\
\hline $\mathrm{Si}$ & $45(50,0)$ & $1(2,2)$ & $5(11,1)$ & $39(86,7)$ & \\
\hline No & $45(50,0)$ & $17(37,8)$ & $26(57,8)$ & $2(4,4)$ & \\
\hline Toma más de tres medicamentos & & & & & $0,033^{* *}$ \\
\hline $\mathrm{Si}$ & $25(27,9)$ & $7(28,0)$ & $9(36,0)$ & $9(36,0)$ & \\
\hline No & $65(72,2)$ & $34(52,3)$ & $22(33,8)$ & $9(13,8)$ & \\
\hline Estrés psicológico & & & & & $<0,0001^{* *}$ \\
\hline $\mathrm{Si}$ & $24(26,7)$ & $8(33,3)$ & $16(66,7)$ & -- & \\
\hline No & $66(73,3)$ & $10(15,1)$ & $15(22,7)$ & $41(62,1)$ & \\
\hline Movilidad & & & & & $<0,0001^{* *}$ \\
\hline No se levanta & $2(2,2)$ & $2(100,0)$ & -- & -- & \\
\hline Levanta no sabe & $44(48,9)$ & $16(36,4)$ & $28(63,6)$ & -- & \\
\hline Puede salir & $44(48,9)$ & -- & $3(6,8)$ & $41(93,2)$ & \\
\hline Problemas neurosicológicos & & & & & $<0,0001^{* *}$ \\
\hline Severo & $1(1,1)$ & $1(100,0)$ & -- & -- & \\
\hline Moderado & $34(37,8)$ & $11(32,3)$ & $22(64,7)$ & $1(2,9)$ & \\
\hline Sin problemas & $55(61,1)$ & $6(10,9)$ & $9(16,6)$ & $40(72,7)$ & \\
\hline Lesiones en piel & & & & & $0.086^{* *}$ \\
\hline $\mathrm{Si}$ & $3(3,3)$ & $2(66,7)$ & $1(33,3)$ & -- & \\
\hline No & $87(96,7)$ & $16(18,4)$ & $30(34,5)$ & $41(47,1)$ & \\
\hline
\end{tabular}

$\alpha=0.05 *$ Test exacto Fisher

Tabla 4. Descripción de la condición nutricional al considerar la valoración nutricional dietética subjetiva del MNA

\begin{tabular}{|c|c|c|c|c|c|}
\hline Variable & $\begin{array}{l}\text { Global } \\
\text { n (\%) }\end{array}$ & $\begin{array}{c}\text { Desnutrido } \\
\text { n (\%) }\end{array}$ & $\begin{array}{c}\text { Riesgo de desnutrición } \\
\mathrm{n}(\%)\end{array}$ & $\begin{array}{l}\text { Bien nutrido } \\
\text { n (\%) }\end{array}$ & $\mathbf{P}^{*}$ \\
\hline Comidas diarias & & & & & $0,200^{*}$ \\
\hline Dos al día & $1(1,1)$ & $1(100,0)$ & $0(0,0)$ & $0(0,0)$ & \\
\hline Tres al día & $89(98,9)$ & $17(19,1)$ & $31(34,8)$ & $41(46,1)$ & \\
\hline \multicolumn{6}{|l|}{ Nivel de proteínas } \\
\hline Cero a una proteínas & $31(34,4)$ & $12(38,7)$ & $18(58,1)$ & $1(3,3)$ & $<0,0001^{*}$ \\
\hline Dos proteínas & $38(42,2)$ & $5(13,2)$ & $12(31,6)$ & $21(55,3)$ & \\
\hline Tres o más proteínas & $21(23,3)$ & $1(4,8)$ & $1(4,8)$ & $19(90,5)$ & \\
\hline Consumo de frutas y verduras & & & & & $0,894^{* *}$ \\
\hline $\mathrm{Si}$ & $82(91,1)$ & $16(19,5)$ & $28(34,1)$ & $38(46,3)$ & \\
\hline No & $8(8,9)$ & $2(25,0)$ & $3(37,5)$ & $3(37,5)$ & \\
\hline Reducción de apetito & & & & & $<0,0001^{*}$ \\
\hline Gran & $2(2,22)$ & $2(100,0)$ & -- & -- & \\
\hline Moderada & $51(56,7)$ & $15(29,4)$ & $27(52,9)$ & $9(17,6)$ & \\
\hline $\operatorname{Sin}$ & $37(41,1)$ & $1(2,7)$ & $4(10,8)$ & $32(86,5)$ & \\
\hline \multicolumn{3}{|c|}{ Consumo de líquido al día (número de tazas) } & & & $<0.0001^{*}$ \\
\hline Menos de tres & $61(67,8)$ & $18(29,5)$ & $25(40,1)$ & $18(29,5)$ & \\
\hline De tres a cinco & $27(30,0)$ & -- & $6(22,2)$ & $21(77,8)$ & \\
\hline Más de cinco & $2(2,2)$ & -- & - & $2(100,0)$ & \\
\hline \multicolumn{2}{|l|}{ Manera de alimentarse } & & & & $<0.0001^{*}$ \\
\hline Incapaz & $2(2,2)$ & $2(100,0)$ & -- & -- & \\
\hline Autoalimenta con dificultad & $17(18,9)$ & $10(58,8)$ & $6(35,5)$ & $1(5,9)$ & \\
\hline Autoalimenta sin dificultad & $71(78,9)$ & $6(8,5)$ & $25(35,2)$ & $40(56,3)$ & \\
\hline \multicolumn{2}{|l|}{ Alteración nutricional } & & & & $<0.0001^{* * *}$ \\
\hline Moderada & $56(62,2)$ & $18(32,1)$ & $30(34,4)$ & $8(14,3)$ & \\
\hline Sin problemas & $34(37,8)$ & -- & $1(2,9)$ & $33(97,1)$ & \\
\hline \multicolumn{2}{|l|}{ Comparación de salud con otros } & & & & $<0.0001^{* * *}$ \\
\hline No tan buena & $15(16,7)$ & $6(40,0)$ & $7(46,7)$ & $2(13,3)$ & \\
\hline No sabe & $35(38,9)$ & $11(31,4)$ & $20(57,1)$ & $4(11,4)$ & \\
\hline Igual de bueno & $27(30,0)$ & $1(3,7)$ & $3(11,1)$ & $23(85,2)$ & \\
\hline Mejor & $13(14,4)$ & -- & $1(7,7)$ & $12(92,3)$ & \\
\hline
\end{tabular}

$\alpha=0.05^{* *}$ Test exacto Fisher

24 


\section{ARTÍCULO ORIGINAL}

Tabla 5. Descripción de la condición nutricional al considerar la valoración del SBU y otros factores orales

\begin{tabular}{|c|c|c|c|c|c|}
\hline Variable & $\begin{array}{l}\text { Global } \\
\text { n (\%) }\end{array}$ & $\begin{array}{c}\text { Desnutrido } \\
\text { n (\%) }\end{array}$ & $\begin{array}{c}\text { Riesgo de desnutrición } \\
\mathbf{n}(\%)\end{array}$ & $\begin{array}{c}\text { Bien nutrido } \\
\text { n (\%) }\end{array}$ & $\mathbf{P}^{*}$ \\
\hline Ardor bucal & & & & & $0,549^{*}$ \\
\hline $\mathrm{Si}$ & $31(34,4)$ & $8(25,8)$ & $9(29,03)$ & $14(45,1)$ & \\
\hline No & $59(65,6)$ & $10(16,9)$ & $22(37,3)$ & $27(45,9)$ & \\
\hline Nivel de ardor bucal & $1,8 \pm 2,6$ & $2,3 \pm 2,7$ & $1,6 \pm 2,5$ & $2,6 \pm 2,6$ & $0,691^{* *}$ \\
\hline Medicamentos & & & & & $<0,0001^{* *}$ \\
\hline $\mathrm{Si}$ & $73(81,1)$ & $17(23,3)$ & $30(41,1)$ & $26(35,6)$ & \\
\hline No & $17(18,9)$ & $1(5,9)$ & $1(5,9)$ & $15(88,2)$ & \\
\hline Dificultad para masticar & & & & & $0.009 *$ \\
\hline SI & $40(44,4)$ & $11(27,5)$ & $18(45,0)$ & $11(27,50)$ & \\
\hline No & $50(55,6)$ & $7(14,0)$ & $13(26,0)$ & $30(60,0)$ & \\
\hline Presenta prótesis dental & & & & & $<0,0001^{* *}$ \\
\hline $\mathrm{Si}$ & $69(76,7)$ & $8(11,6)$ & $21(30,4)$ & $40(57,1)$ & \\
\hline No & $21(23,3)$ & $10(47,6)$ & $10(47,6)$ & $1(4,8)$ & \\
\hline Prótesis dental superior & & & & & $<0,0001^{* *}$ \\
\hline $\mathrm{Si}$ & $69(76,7)$ & $8(11,6)$ & $20(29,0)$ & $4(59,4)$ & \\
\hline No & $21(23,3)$ & $10(47,6)$ & $11(52,4)$ & -- & \\
\hline \multicolumn{6}{|l|}{ Prótesis dental inferior } \\
\hline $\mathrm{Si}$ & $28(31,1)$ & $2(7,1)$ & $5(17,9)$ & $21(75,0)$ & $0.086^{* *}$ \\
\hline No & $62(68,9)$ & $16(25,8)$ & $26(41,9)$ & $20(32,3)$ & \\
\hline Prótesis desadaptadas & & & & & $0.873^{* *}$ \\
\hline $\mathrm{Si}$ & $15(16,7)$ & $2(13,3)$ & $6(40,0)$ & $7(46,7)$ & \\
\hline No & $75(83,3)$ & $16(21,3)$ & $25(33,3)$ & $34(45,3)$ & \\
\hline Presencia de candidiasis & & & & & $0.099^{* *}$ \\
\hline $\mathrm{Si}$ & $15(16,7)$ & $3(37,5)$ & $4(50,0)$ & $1(12,5)$ & \\
\hline No & $75(83,3)$ & $15(18,3)$ & $27(32,9)$ & $40(48,8)$ & \\
\hline Boca seca & & & & & $0.267^{*}$ \\
\hline $\mathrm{Si}$ & $50(55,6)$ & $11(22,0)$ & $20(40,0)$ & $19(38,0)$ & \\
\hline No & $40(44,4)$ & $7(17,5)$ & $11(27,5)$ & $22(55,0)$ & \\
\hline Flujo salival & & & & & $0.074^{*}$ \\
\hline Normal & $59(65,6)$ & $10(16,9)$ & $17(28,8)$ & $32(54,2)$ & \\
\hline Anormal & $31(34,4)$ & $8(25,8)$ & $14(45,2)$ & $9(29,03)$ & \\
\hline
\end{tabular}

Tabla 6. Descripción de la condición nutricional al considerar la ingesta de medicamentos

\begin{tabular}{|c|c|c|c|c|c|}
\hline Variable & $\begin{array}{l}\text { Global } \\
\text { n (\%) }\end{array}$ & $\begin{array}{c}\text { Desnutrido } \\
\text { n (\%) }\end{array}$ & $\begin{array}{c}\text { Riesgo de desnutrición } \\
\mathrm{n}(\%)\end{array}$ & $\begin{array}{c}\text { Bien nutrido } \\
\text { n (\%) }\end{array}$ & $\mathbf{P}^{*}$ \\
\hline Número medicamentos & $1,71 \pm 1,1$ & $2,22 \pm 1,1$ & $2,0 \pm 1,0$ & $1,30 \pm 1,2$ & $0,0022^{* *}$ \\
\hline Antisicóticos & $8(8,9)$ & $2(25,0)$ & $5(62,5)$ & $11(12,5)$ & $0,112^{* *}$ \\
\hline Antihistamínico & $51(56,7)$ & $11(21,6)$ & $20(39,2)$ & $20(39,2)$ & $0,375^{*}$ \\
\hline Diurético & $26(28,9)$ & $6(23,1)$ & $12(49,1)$ & $8(30,8)$ & $0,184^{*}$ \\
\hline Glucosamida & $2(2,2)$ & ----- & -.--- & $2(100,0)$ & $0,683^{* *}$ \\
\hline Broncodilatadora & $1(1,1)$ & ----- & $1(100,0)$ & ----- & $0,544^{* *}$ \\
\hline Analgésicos & $4(4,4)$ & $1(25,0)$ & ----- & $3(75,0)$ & $0,332^{* *}$ \\
\hline Tiroideos & $3(3,3)$ & $3(100,0)$ & ------ & ------ & $0,007^{* *}$ \\
\hline Antimicóticos & $2(2,2)$ & $1(50,0)$ & $1(50,0)$ & ------ & $0,294^{* *}$ \\
\hline Hipoglicemiantes & $14(15,6)$ & $7(50,0)$ & $3(21,4)$ & $4(28,6)$ & $0,017^{* *}$ \\
\hline Antiinflamatorios & $4(4,4)$ & $1(25,0)$ & $3(75,0)$ & ------ & $0,096^{* *}$ \\
\hline Ansiolíticos & $16(17,8)$ & $1(6,2)$ & $8(50,0)$ & $7(43,7)$ & $0,210^{* *}$ \\
\hline Corticoides & $16(17,8)$ & $6(37,5)$ & $5(31,2)$ & $5(31,5)$ & $0,141^{*}$ \\
\hline Antihistamínicos & $7(7,8)$ & $1(14,3)$ & $4(57,1)$ & $2(28,6)$ & $0,530^{* *}$ \\
\hline
\end{tabular}

$\alpha=0.05^{*}$ Test Chi ${ }^{2 *}$ Test exacto Fisher 
ARTÍCULO ORIGINAL

Tabla 7. Descripción de condición nutricional al considerar la disgeusia

\begin{tabular}{|c|c|c|c|c|c|}
\hline Variable & $\begin{array}{l}\text { Global } \\
\text { n (\%) }\end{array}$ & $\begin{array}{l}\text { Desnutrido } \\
\text { n (\%) }\end{array}$ & $\begin{array}{c}\text { Riesgo de desnutrición } \\
\mathrm{n}(\%)\end{array}$ & $\begin{array}{c}\text { Bien nutrido } \\
\mathbf{n}(\%)\end{array}$ & $\mathbf{P}^{*}$ \\
\hline Autoreporte de disgeusia & & & & & $0.210^{* *}$ \\
\hline $\mathrm{Si}$ & $87(96,7)$ & $17(19,5)$ & $29(33,3)$ & $41(47,1)$ & \\
\hline No & $3(3,3)$ & $1(3,3)$ & $2(66,8)$ & -- & \\
\hline Alteración del sabor & & & & & $0.499^{*}$ \\
\hline $\mathrm{Si}$ & $66(73,3)$ & $13(19,7)$ & $25(37,9)$ & $28(42,4)$ & \\
\hline No & $24(26,7)$ & $5(20,8)$ & $6(25,0)$ & $13(54,2)$ & \\
\hline Dulce & & & & & $0.423^{* *}$ \\
\hline Ninguno & $39(43,3)$ & $8(20,5)$ & $16(41,0)$ & $15(38,5)$ & \\
\hline Poco & $18(20,0)$ & $5(27,8)$ & $5(27,8)$ & $8(44,4)$ & \\
\hline Normal & $18(20,0)$ & $1(5,6)$ & $5(27,8)$ & $12(66,7)$ & \\
\hline Muy & $15(16,7)$ & $4(26,7)$ & $5(33,3)$ & $6(40,0)$ & \\
\hline Salado & & & & & $0.110^{* *}$ \\
\hline Ninguno & $17(18,9)$ & $5(29,4)$ & $7(41,2)$ & $5(29,4)$ & \\
\hline Poco & $42(46,7)$ & $6(14,3)$ & $11(26,2)$ & $25(59,5)$ & \\
\hline Normal & $17(18,9)$ & $1(5,9)$ & $7(41,2)$ & $9(52,9)$ & \\
\hline Muy & $14(15,6)$ & $6(42,9)$ & $6(42,9)$ & $2(14,3)$ & \\
\hline Ácido & & & & & $0.024^{* *}$ \\
\hline Ninguno & $16(17,8)$ & $7(43,8)$ & $6(37,5)$ & $3(18,7)$ & \\
\hline Poco & $49(54,4)$ & $6(12,2)$ & $14(28,6)$ & $29(59,2)$ & \\
\hline Normal & $22(24,4)$ & $5(22,7)$ & $10(45,4)$ & $7(31,8)$ & \\
\hline Muy & $3(3,3)$ & -- & $1(33,3)$ & $2(66,7)$ & \\
\hline Amargo & & & & & $0.110^{* *}$ \\
\hline Ninguno & $21(23,3)$ & $1(4,8)$ & $7(33,3)$ & $13(61,9)$ & \\
\hline Poco & $39(43,3)$ & $10(25,6)$ & $16(41,0)$ & $13(33,3)$ & \\
\hline Normal & $23(25,6)$ & $7(30,4)$ & $6(26,1)$ & $10(43,5)$ & \\
\hline Muy & $7(7,8)$ & -- & $2(28,6)$ & $5(71,4)$ & \\
\hline
\end{tabular}

\section{Análisis bivariado}

\section{Variables sociodemográficas}

Se observaron asociaciones estadísticamente significativas para género $(p=0,010)$, edad $(p=0,002)$, vinculación institucional $(\mathrm{p}<0,0001)$ y nivel de escolaridad $(\mathrm{p}<0,0001)$. Es importante anotar que mientras el $29,7 \%$ (11) de los hombres registraron una condición de desnutrición sólo el 13,2\% (7) de las mujeres lo reportaron. En relación con la edad, se encontró que las personas desnutridas registraron un promedio de edad de 73,8 \pm 8,3 años; los bien nutridos reportaron un promedio de 66,5 \pm 7,4 años.

En relación con la vinculación institucional se destacó que mientras el 39,1\% (18) de los institucionalizados registraron desnutrición en ninguno de los no institucionalizados se observó esta condición. En cuanto al nivel de escolaridad se destacó que del total de aquellos que reportaron no tener algún nivel educativo el 41,9\% (13) registró desnutrición;
9,4\% (5) tenían un nivel primario y ninguno de los de nivel secundario u otros reportaron desnutrición (Tabla 1).

\section{Variables antropométricas del MNA}

Se observaron asociaciones estadísticamente significativas para Índice de Masa Corporal (IMC) $(\mathrm{p}<0,0001)$, pérdida de peso $(\mathrm{p}<0,0001)$, peso $(\mathrm{p}<0,0001)$ y circunferencia braquial $(\mathrm{p}<0,0001)$. Se encontró que las personas desnutridas registraban un promedio de $47,4 \pm 10,6 \mathrm{Kg}$ al compararlo con los bien nutridos que registraron un promedio de $64,8 \pm 11,1 \mathrm{Kg}$. Para el Índice de Masa Corporal (IMC) es importante anotar que el 55,6\% (10) del total de personas con un IMC entre 19 a $21 \mathrm{Kg} /$ $\mathrm{m}^{2}$ registraron desnutrición y del total de personas con IMC menor a 21 el 40,0\% (4) presentaron desnutrición. Así mismo, del total de personas que no saben si han tenido pérdida de peso en los últimos tres meses, el 28,6\% (18) registró desnutrición. 
Adicionalmente, se observó que del total de personas con una circunferencia braquial mayor a 22 el $3,1 \%$ (2) registró desnutrición. Al revisar la pérdida de peso es importante destacar que del total de personas que reportaron que no había pérdida de peso, el $100 \%$ estaba bien nutridos mientras que del total que no sabían el 28,6 \% (18) estaban bien nutridos (Tabla 2).

\section{Variables correspondientes a la valoración global del MNA}

Para este conjunto de variables se observaron asociaciones estadísticamente significativas excepto para lesiones en la piel. El 37,8\% (17) de las personas que no vivían de manera independiente registró una condición de desnutrición mientras que el $2,2 \%$ (1) de los que sí lo hacía reportó una condición igual. El 52,3\% (34) de las personas que no tomaban más de tres medicamentos presentaron una condición de desnutrición mientras que el 28,0\% (7) de las personas que consumían más de tres medicamentos no registraron igual condición.

En relación con la varibale estrés psicológico ( $p<0,0001$ ), el 33,3\% (8) de las personas que habían tenido estrés psicológico registraron desnutrición y sólo el 15,1\% (10) de las personas que no registraron estrés psicológico estaban desnutridos. Es importante resaltar que del total de personas que podían salir, el 93,2\% (41) tenían una buena condición nutricional. En cuanto a los problemas neuropsicológicos, el 100,0\% (1) de las personas con demencia severa presentaron desnutrición, sólo el 32,3\% (11) con demencia moderada registró igual condición y el 10,9\% (6) de las personas sin problemas neuropsicológicos reportó desnutrición. El 66,7\% (2) de las personas que presentó lesiones de piel registraron desnutrición, sólo el 18,4\% (16) de las personas sin lesiones de piel reportó igual condición (Tabla 3).

\section{Variables correspondientes a valoración dietética y subjetiva}

No se encontraron asociaciones estadísticamente significativas para ingestas diarias $(p=0,2)$ y consumo de frutas o verduras $(p=0,894)$. Con respecto al consumo de proteínas se observó que del total de personas que consumían entre cero y una proteína $(\mathrm{p}<0,0001)$ al día, el 38,7\% (12) estaba desnutrido y sólo el 4,8\% (1) de las que reportaban consumir tres o más proteínas registró igual condición.

En relación con la pérdida del apetito el 100\% (2) de las personas con gran pérdida de apetito estaban desnutridas mientras que sólo el 2,7\% (1) de las que no reportaron pérdida de apetito registraron igual condición. Para el consumo de agua y otros líquidos $(p<0.0001)$ se encontró que del total de personas que consumían menos de tres tazas estaban desnutridos y ninguna de las que consumían tres o más tazas registraron esta condición.

Con respecto a la manera de alimentarse de las personas que reportan incapacidad para autoalimentarse el 100\% (2) están desnutridas mientras que las que reportan autoalimentarse sin dificultad el $8,5 \%$ (6) registra algún nivel de desnutrición.

Del total de personas que se auto perciben con una condición de desnutrición el 32,1\% (18) está desnutrido y ninguno de los que reportan no auto percibir problemas está desnutrido. Al comparar su estado de salud con el de otras personas $(\mathrm{P}<0,0001)$ del total de personas que consideran que su estado de salud no es tan bueno el 40\% (6) está desnutrido y sólo del total de personas que consideran su estado de salud igual al de otros el 3,7\% (1) registra condición de desnutrición (Tabla 4).

\section{Variables relacionadas con Síndrome de Boca Urente (SBU) y otros factores orales}

Se registraron diferencias estadísticamente significativas para las variables medicación $(\mathrm{p}<0,001)$, dificultad para masticar $(p=0,009)$, presencia de prótesis superior $(\mathrm{p}<0,0001)$, presencia de prótesis inferior $(\mathrm{p}=0,001)$.

Del total de personas que registraron medicación, el $23,3 \%$ (17) estaba desnutrida y sólo el 5,9\% (1) de las que no registraron medicación mostraba una condición de desnutrición. Del total de personas que no presenta prótesis dental o prótesis superior, el $47,6 \%$ ( 10 ) reportó desnutrición y sólo el $11,6 \%$ (8) de las que tenían prótesis estaba desnutrida.

Del total de personas que no registran prótesis inferior, el 25,8\% (16) está desnutrida y sólo el 7,1\% (2) de las que presentan este tipo de rehabilitación registran desnutrición.

Es importante destacar que al referirnos al SBU que del total de personas que lo registran el 25,8\% (8) está desnutrido y sólo el $16,9 \%$ (10) de las que no lo presentan registran condición de desnutrición con un promedio de intensidad de ardor de $2,3 \pm 2,71$ en las personas que registran desnutrición (Tabla 5).

\section{Variables relacionadas con tipo de medicamentos}

$\mathrm{Al}$ analizar los medicamentos relacionados con la medicación se encontró que las variables número de medicamentos, consumo de medicamentos para control de alteraciones tiroideas y consumo de hipoglicemiantes se asociaron en forma estadísticamente significativas con la condición nutricional.

$\mathrm{Al}$ referirse al número de medicamentos se observa que el promedio de medicamentos consumidos por 
las personas desnutridas es de 2,22 $\pm 1,11$ comparado con las bien nutridas que es de $1,3 \pm 1,20$. Al referirse al consumo de medicación para el manejo de condiciones tiroideas se observa que del total de personas que consumen este tipo de medicamentos todos están bajo la condición de desnutrición y al referirse a hipoglicemiantes se encuentra que del total de personas que consumen este tipo de medicamentos el 50\% (7) están desnutridos y sólo el 14,5\% (11) de los que no lo consumen registra desnutrición (Tabla 6).

\section{Variables relacionadas con disgeusia}

$\mathrm{Al}$ referirse a aspectos relacionados con la disgeusia es importante anotar que del total de personas que no perciben los sabores ácidos el 43,8\% (7) está desnutrido y sólo el $22,7 \%$ (5) de los que perciben de forma normal este sabor reporta una condición nutricional anormal por lo cual reencuentran asociaciones estadísticamente significativas con este sabor percibido $(P=0,024)$.

Se destaca, además, que del total de personas que no tienen alteración en el sabor el 54,2\% (13) está bien nutrido comparado con el 20,8\%(5) que si registra desnutrición (Tabla 7).

\section{Análisis multivariado}

En el análisis de regresión binomial no ajustado, fueron varias las variables que se asociaron significativamente con desnutrición o a riesgo en el grupo de adultos mayores evaluados, dentro de las que cabría destacar vive independiente (RP 19,5 IC 95\% 5,0 $75,9)$, trastornos neurosicológicos (RP 25,5 IC 95\% 3,7 176,8 ) y consumo de proteínas (RP 28 IC 95\% 4,1 193), entre otras. Por su parte, la variable sabor amargo pareció comportarse como un factor protector con una RP de 0,5 (IC 95\% 0,3 0,9) (Tabla 8).

En el modelo de regresión binomial ajustado, las variables que permanecieron como estadísticamente significativas a la desnutrición en los adultos mayores evaluados fue vive independiente (RP 8,3 IC 95\% $1,883,3)$, y dentro de las variables relacionados con la condición oral estuvieron dificultad para masticar y boca seca ambas con RP 1,4 (IC 95\% 1,2 1,7). Sabor amargo continuó comportándose como factor protector con una RP 0,7 (IC 95\% 0,3 0,9) (Tabla 9).

Tabla 8. Análisis de regresión binomial no ajustado de las variables evaluadas en la población adulta mayor asociadas con la desnutrición

\begin{tabular}{|c|c|c|c|}
\hline Variable & $\mathbf{R P}^{*}$ & IC 95\%** & $\mathbf{P}$ \\
\hline Hombre & 2,2 & $1,23,8$ & 0,009 \\
\hline Mayor de 70 años & 3,1 & $1,65,7$ & $<0,0001$ \\
\hline Ninguna escolaridad & 6,7 & $2,219,8$ & 0,001 \\
\hline No vive independiente & 19,5 & $5,075,9$ & $<0,0001$ \\
\hline Más de tres medicamentos & 1,9 & $0,93,6$ & 0,001 \\
\hline Trastorno neuropsicologico & 25,5 & $3,7176,8$ & 0,001 \\
\hline 0-1/2 proteínas & 17,1 & $2,4120,3$ & 0,004 \\
\hline 0-1/3 proteínas & 28,0 & $4,1193,0$ & 0,001 \\
\hline$<3 / 3-5$ tazas & 2,6 & $1,74,1$ & $<0,0001$ \\
\hline SBU & 1,0 & $0,42,5$ & 0,957 \\
\hline Dificultad para masticar & 2,2 & $1,33,8$ & 0,006 \\
\hline Presencia prótesis & 12,2 & $1,883,3$ & 0,011 \\
\hline Prótesis desadaptada & 1,0 & $0,61,9$ & 0,924 \\
\hline Boca seca & 1,4 & $0,92,3$ & 0,109 \\
\hline Flujo salival disminuido & 1,9 & $1,03,4$ & 0,041 \\
\hline Alteración del sabor & 0,8 & $0,51,2$ & 0,301 \\
\hline No percibe salado & 2,0 & $0,94,4$ & 0,076 \\
\hline No percibe ácido & 3,2 & $1,19,0$ & 0,031 \\
\hline No percibe amargo & 0,5 & $0,30,9$ & 0,029 \\
\hline No percibe dulce & 1,2 & $0,62,2$ & 0,664 \\
\hline Poco percibe dulce & 1,7 & $1,02,9$ & 0,036 \\
\hline
\end{tabular}

*Razón de prevalencia ** Intervalo de confianza del 95\% 
Tabla 9. Análisis de regresión binomial ajustado de las variables evaluadas en la población adulta mayor asociadas con la desnutrición

\begin{tabular}{llccc}
\hline & Variable & $\mathbf{R P}^{*}$ & IC 95\%** & P \\
\hline Hombre & 1,0 & 1,0 & 1,0 & $<0,0001$ \\
No vive independiente & 8,3 & 2,1 & 32,8 & 0,002 \\
Trastorno neuropsicológico & 4,9 & 0,9 & 28,2 & 0,074 \\
Dificultad para masticar & 1,4 & 1,2 & 1,7 & $<0,0001$ \\
Prótesis desadaptada & 1,1 & 0,9 & 1,2 & $<0,0001$ \\
Boca seca & 1,2 & 1,7 & $<0,0001$ \\
No percibe amargo & 1,4 & 0,6 & 0,6 & $<0,0001$ \\
No percibe dulce & 0,7 & $1,1<1,1$ & $<0,0001$ \\
\hline
\end{tabular}

*Razón de prevalencia ** Intervalo de confianza del 95\%

\section{DISCUSIÓN}

El mantenimiento del equilibrio de la función oral depende de la sensación y de las experiencias de cada persona en relación con su la cavidad bucal. El envejecimiento va acompañado de diversos cambios fisiológicos, psicológicos, económicos y sociales que pueden afectar el estado nutricional y aun cuando la desnutrición no es una consecuencia inevitable del envejecimiento, con ésta se producen cambios que pueden contribuir a su génesis. ${ }^{10}$

En este trabajo no se encontró asociación del SBU y disgeusia con los trastornos nutricionales. Aunque si se logró establecer una asociación entre el sabor ácido con los trastornos nutricionales. Así mismo, se observó que el 39,1\% de las personas alojadas en el hogar geriátrico registraron desnutrición mientras que en las personas que residían en su propia vivienda no se observó esta condición. Diferentes situaciones y factores (ambientales, funcionales, psicológicas, nutricionales y médicas), pueden llevar a que el adulto mayor disminuya su ingesta de alimentos lo que contribuye al desarrollo de malnutrición. En adultos mayores institucionalizados el estado nutricional se ve afectado principalmente por factores nutricionales que se asocian positivamente con disfagia, consumo lento de alimentos, ingesta proteica baja, apetito disminuido y la edad. ${ }^{10}$ En este trabajo se encontró una disminución en el consumo de proteínas en el 38,7\% de las personas evaluadas.

Por otra parte, el peso varía de acuerdo con la edad y el sexo de la persona. En los países desarrollados, el peso de hombres y mujeres se incrementa a la edad media (50-65 años); los incrementos de los hombres tienden a estabilizarse alrededor de los 65 años y disminuyen después de esta edad. En las mujeres esta estabilización se presenta diez años después y el aumento de peso es mayor que en el de los hombres. ${ }^{20}$ En este estudio se encontró que el 29,7\% de los hombres registraron desnutrición y sólo el $13,2 \%$ de las mujeres la presentaba lo que parece relacionarse con lo ya mencionado. Sin embargo, este resultado no coincide con los presentados por Peña y colaboradores quienes identificaron una prevalencia de malnutrición de 58,0\% para el género femenino y 2,20 \% para el masculino. ${ }^{10,22}$ La ausencia de un instrumento específico validado para evaluar el estado nutricional de las personas ancianas podría explicar la inexistencia, hasta fechas recientes, de una valoración geriátrica exhaustiva. ${ }^{20,23,24}$ Así pues, en los adultos mayores, la valoración del estado de nutrición es especialmente difícil porque muchos de los signos relacionados con la desnutrición son signos del proceso de envejecimiento. En cualquier caso la valoración tiene cuatro componentes: antecedentes nutricionales y evaluación dietética, indicadores antropométricos, exploración física y pruebas de laboratorio. Ninguno de los cuatro componentes es definitivo lo que significa que un resultado sin el otro no aporta información completa de la situación nutricional de la persona. Es así como desde hace un tiempo se ha hecho hincapié en el desarrollo y validación de instrumentos eficaces para detectar riesgos de malnutrición o situaciones de malnutrición evidentes, puesto que una vez que ha aparecido la desnutrición su corrección es difícil y su pronóstico desfavorable y por ello la actuación de los profesionales de la salud debe estar dirigida hacia estrategias preventivas menos costosas y más fáciles de llevar a cabo. ${ }^{23}$

Celestino y colaboradores encontraron que en relación con el riesgo de malnutrición medido por el MNA, se obtuvieron los siguientes resultados: el $58,8 \%$ (50) se ubicó dentro de un buen estado de nutrición ( $>23,5$ puntos), el 40\% presentó riesgo de malnutrición ( $<17$ puntos) y el $1,2 \%$ (2) se encontró en la categoría de malnutrición. ${ }^{25}$ En el presente estudio se identificó que del total de participantes 
el 20\% (18) presentó desnutrición, el 34,4\% (3) se encontraba a riesgo de desnutrición y el $45,56 \%$ (41) presentó una condición nutricional adecuada. En este trabajo no se contó con la evaluación de una nutricionista y aunque el instrumento usado ha sido validado en otras poblaciones, en ésta no se realizó la validación. Estos aspectos podrían considerarse como limitaciones del trabajo.

De acuerdo con la literatura, el ardor bucal ocurre más en mujeres que en hombres en una relación aproximada de $3: 1$ a 9:1. ${ }^{26}$ No hay datos establecidos sobre su prevalencia debido a la variación que se ha reportado en los diferentes estudios. En el presente estudio se observó un promedio de edad de 70,4 $\pm 8,6$ años y se identificó que el $55,6 \%$ de casos reportaron sensación de boca seca. La proporción es muy similar a la que reportaron Grushka y colaboradores, quienes informaron una prevalencia de xerostomia superior al $60 \%,{ }^{18}$ además se encontraron diferencias estadísticamente significativas para la variable medicación, dificultad para masticar alimentos y presencia de prótesis dental.

Se sabe que uno de los principales factores que pueden influir con el ardor bucal es el consumo de medicamentos, algunos de los más importantes son los psicotropos, los antihipertensivos y los diuréticos; ${ }^{27}$ en el presente estudio se identificó que el 56,7\% (51) de las personas evaluadas consumía antihistamínicos, el 28,9\% (26) diuréticos, el 15,6\% (14) hipoglicemiantes y el $17,8 \%$ (16) ansiolíticos y corticoesteroides.

En el presente trabajo se evidenció que en el adulto mayor el umbral gustativo para cada uno de los sabores básicos presentaba una reducción importante respecto a los valores que la literatura reporta como normales. ${ }^{25} \mathrm{Al}$ comparar los valores obtenidos en el adulto mayor respecto a los umbrales gustativos de los individuos jóvenes, se encontraron diferencias estadísticamente significativas que mostraron una reducción fisiológica de este promedio sensorial, aunque este resultado no se encontró en el sabor ácido. Cabe mencionar que estos hallazgos no coinciden con los obtenidos por Gonzáles - Carnero (2002), quienes reportaron una reducción significativa del umbral gustativo únicamente para los sabores dulce y salado en los adultos mayores ${ }^{28}$.

El sentido del gusto y el olfato son en gran parte, responsables de la selección de los alimentos en las personas por lo que al estar relacionados con los hábitos dietéticos podrían influir directa o indirectamente sobre el estado nutricional. Por lo tanto, comprender que la mayoría de los supuestos achaques que padecen los adultos mayores no son atribuidos a procesos patológicos, sino a respuestas humanas de índole fisiológico atribuibles al envejecimiento permitirá que los profesionales ofrezcan un servicio de mayor calidad y calidez ${ }^{17,}$ 28-31. Un limitante encontrado en este estudio fue la no evaluación de la percepción de los olores de cada paciente.

La intención inicial de esta investigación fue evaluar el SBU pero hubo dificultad en el proceso de diagnóstico por lo cual se trabajó con el ardor bucal. Se sugiere realizar próximas investigaciones que faciliten el diagnóstico de SBU al considerar que esta patología es muy frecuente en adultos mayores.

\section{Conclusiones}

El ardor bucal no se asoció con las alteraciones nutricionales en los adultos mayores evaluados. En cuanto a la disgeusia, la alteración en la percepción del sabor amargo se comportó como un factor protector con RP 0,7 (IC 95\% 0,3 0,9).

El 34,5 \% del total de las personas evaluadas reportaron sufrir de ardor bucal.

El 73,3\% del total de los adultos mayores registraron disgeusia y de ellos el 43,3\% (39) reportaron alteración en la percepción del sabor dulce y el $23 \%$ (21) del sabor amargo.

Las variables que se asociaron con el estado de desnutrición o riesgo de desnutrición (de acuerdo al MNA) fueron vive independiente (RP 8,3 IC 95\% 1,8 83,3) y dentro de los factores orales dificultad para masticar y boca seca, ambos con RP 1,4 (IC 95\% 1,2 1,7).

\section{BIBLIOGRAFÍA}

1. Soto M, Alcallaga RG, Esguep A. Asociación entre alteraciones psicológicas y la presencia de liquen plano oral, síndrome de boca urente y estomatitis aftosa recibidante. Med Oral 2004; 9: 1 - 7.

2. Barrera S, Sacsoquispe S, Barela L. Frecuencia del síndrome de boca urente y factores asociados en adultos mayores. Diagnóstico (Perú) 2006; 45: 64 - 79.

3. Donat S, Serrano MF. El síndrome de boca urente, revisión de conceptos y puesta al día. Medicina Oral 1997; 2: 30 - 38 .

4. Palacios M, Jordana XC, Gracia SC. Burning Mouth Syndrome. A retrospective study of 140 cases in a simple of Catalan population. Med Oral Patol Oral Cir Bucal 2005; 10: $388-393$

5. Epstein S. Importance of psychosocial and behavioral factors in food ingestion in the elderly and their ramifications on oral health. Gerodontics 1987: 3: $23-25$.

6. Lamster IB. Oral health care services for older adults: a looming crisis. Am J Public Health 2004; 94: 699 - 702.

7. Ralph J, Pearson JR. Burning Mouth- a case report. Gerodontics 1988: 4: 41 - 42.

8. Grushka M, Epstein JB, Gorsky M. Burning mouth syndrome. Am Fam Physician 2002; 65: 615 - 620.

9. Rodríguez de Rivera ME, López J, Chimenos E, Sabater MM. Estudio de una muestra de pacientes con síndrome 
de boca ardiente. Av Odontoestomatol 2007; 23: 141 151.

10. Rodríguez N, Hernández R, Herrera H, Barbosa J, Hernández VY. Estado nutricional de adultos mayores institucionalizados venezolanos. Invest Clin 2005; 46. URL disponible en: http://www.scielo.org.ve/scielo. php?pid $=$ S0535-51332005000300003\&script $=$ sci_art text

11. López C, Bejarano D, Jiménez MD, Mamian Z, Morales FL, Calzada MT, Barreto JF. Influencia de dos técnicas de higiene lingual en el sentido del gusto en ancianos de cuatro hogares geriátricos de Cali. Institución: Universidad del Valle. Septiembre de 2009. URL disponible en. http://salud.univalle.edu.co/pdf/simpsios/octavo/microbiologia/influencia de dos tecnicas de higiene lingual_en_el_sentid.pd̄f

12. Gutiérrez JG, A. Serradle Z, Guevara C. Prevalencia de desnutrición del adulto mayor al ingreso hospitalaria. Nutr Hosp. 2007; 22: 702 - 709.

13. Becerra F. Tendencias actuales en la valoración antropométrica del anciano. Revista Facultad de Medicina de la Universidad Nacional de Co_lombia 2006; 54.

14. Ruiz-López M, Artacho M, López MC. Recomendaciones nutricionales para los ancianos. Ars Pharmaceutica, 2000, 41: $101-113$.

15. Sandoval L, Varela L. Evaluación del estado nutricional de pacientes adultos mayores ambulatorios. Rev Med Hered 1998; 9: $104-108$.

16. Araújo GJ, Jiménez VL, Benítez J. Escala para identificar desnutrición energético- proteica del adulto mayor hospitalizado. Rev Med IMSS 2004; 42 (5): 387 - 394.

17. Bromley SM. Smell and taste disorders: a primary care approach. Am Fam Physician 2000; 61: 427 - 436.

18. Grushka M, Bartoshuk LM. Oral dysesthesias and burning mouth syndrome: taste is a piece of the puzzle. Cand Diagnosis J 2000; 99 - 109.

19. Olivas-Gasteum R, Nevárez-Moorillon GV, Gasteum-Franco MG. Las pruebas de diferencia en el análisis de alimentos. Tecnociencia [en línea] 2009. 8(1): 1 - 7. URL disponible en: http://tecnociencia.uach.mx/numeros/v3n1/data/ AnalisisSensorialdeAlimentos.pdf

20. Becerra F. Tendencias actuales en la valoración antropométrica del anciano. Rev Fac Med Unal 2006; 54: 283 289.

21. Pagano M, Gauvreau K. Fundamentos de Bioestadística. Ed. Thomson Learning 2001. p. 259 -321.

22. Ministerio de Salud. Resolución No. 008430. Por la cual se establecen las normas científicas, técnicas y administrativas para la investigación en salud (octubre de 1993). Bogotá.

23. Falque L, Maestre GE, Zambrano R, Morán de Villalobos Y. Deficiencias nutricionales en los adultos y adultos mayores. An Venez Nutr [en línea] 2005; 18: 82-9. URL disponible en: http://www.scielo.org.ve/scielo.php?script=sci arttext\&pid $=$ S0798-07522005000100016\&lng $=e s$

24. Barrera MP, Castillo MP, Martín J, Torrado J, Cifuentes AM, Rodríguez MN. Evaluación nutricional de adultos mayores hospitalizados en la clínica Carlos Lleras Restrepo de Bogotá D.C utilizando un instrumento adaptativo del Mini Nutritional Assessment (MNA). Rev Asoc Colomb Gerontol Geriatr 2008: 22: 1163 - 1172 .

25. Celestino-Soto MI, Salazar-González BC, Novelo-Huerta HI. Nutrición y desempeño cognitivo del adulto mayor. Universidad de la Sabana, Aquichan [en línea] 2008; 8:
159-169. URL disponible en: http://aquichan.unisabana. edu.co/index.php/aquichan/article/view/133

26. Perdomo M, Chimenos E. Síndrome de Boca Ardiente: actualización. Av Odontoestomatol 2003; 19-4: 193-202

27. Feldblum Ll, Larisa G, Castel H, Harman Ll, Bilenko N, Eisinger M, Fraser D. Characteristics of undernourished older medical patients and the identification of predictors for under nutrition status. Nutr J 2007; 6: 37.

28. Velasco R, Del Toro M, Mora AB, Olmedo BA, Delgado de la Cruz M, Cobián AC. Cuantificación del umbral gustativo del adulto mayor y su comparación con el joven. Rev Cubana Invest Biomed [en línea] 2008; 27 (3-4). URL disponible en: http://www.imbiomed.com.mx/1/1/articulos.php? method $=$ showDetail\&id_articulo $=55528 \&$ id seccion $=666 \&$ id ejemplar $=5624 \&$ id revista $=67$

29. Kalu DN, Masoro EJ. Metabolic and nutritional aspects of aging. Gerodontics 1986: 2: 121 - 126.

30. Brailo V, Vueivi-Boras V, Alajbeg IZ, Alajbeg I, Lukenda J, Eurkovi M. Oral burning symptoms and burning mouth syndrome-significance of different variables in 150 patients. Med Oral Patol Oral Cir Bucal 2006; 11: E252 E255.

31. Nakazato Y, Ito Y, Naito S, Tamura N, Shimazu K. Disgeusia limited to sweet taste in myasthenia gravis. Intern Med 2008; 47: 877 - 878.

\section{Correos electrónicos de los autores:}

Lizeth Johana Muñoz Pérez: lisethe 01@hotmail.com

Diana Riátiga Ortiz: sanandreseā@hotmail.com Sonia Constanza Concha Sánchez: sococosa@yahoo.com 